\title{
Risk factors for sporadic listeriosis: A systematic review and meta-analysis
}

\author{
Alexandre Leclercq ${ }^{\mathrm{a}, 1}$, Pauline Kooh ${ }^{\mathrm{b}, *, 1}$, Jean-Christophe Augustin ${ }^{\mathrm{c}}$, Laurent Guillier ${ }^{\mathrm{b}}$, \\ Anne Thébault ${ }^{\mathrm{b}}$, Vasco Cadavez ${ }^{\mathrm{d}}$, Ursula Gonzales-Barron ${ }^{\mathrm{d}}$, Moez Sanaa ${ }^{\mathrm{b}}$ \\ ${ }^{a}$ Institut Pasteur, National Reference Centre and WHO Collaborating Centre for Listeria, Paris, France; Institut Pasteur, Biology of Infection Unit, Paris, France \\ ${ }^{\mathrm{b}}$ Risk Assessment Department, French Agency for Food, Environmental and Occupational Health \& Safety (Anses), Maisons-Alfort, France \\ ${ }^{\mathrm{c}}$ Ecole Nationale Vétérinaire d'Alfort, Maisons-Alfort, France \\ ${ }^{\mathrm{d}}$ Centro de Investigação de Montanha (CIMO), Instituto Politécnico de Bragança, Campus de Santa Apolónia, 5300-253 Bragança, Portugal
}

\section{A R T I C L E I N F O}

\section{Keywords:}

Listeria monocytogenes

Research synthesis

Case-control studies

Observational studies

\begin{abstract}
A B S T R A C T
Listeriosis is a major public health concern associated with high hospitalization and mortality rates. The objective of this work was to summarize evidence on the associations between risk factors and sporadic cases by meta-analysing outcomes from currently published case-control studies. Suitable scientific articles were identified through systematic literature search, and subjected to a methodological quality assessment. From each study, odds-ratio (OR) measures as well as study characteristics such as population type, design, type of model and risk factor hierarchy were extracted. Mixed-effects meta-analysis models were adjusted by population type to appropriate data partitions.

Twelve primary studies investigating sporadic listeriosis conducted between 1985 and 2013 passed through a quality assessment stage. These studies provided 226 OR considered for meta-analysis.

According to the meta-analysis, the main risk factor for acquiring listeriosis is suffering from an immunocompromising disease. In relation to the food exposures, this meta-analysis confirmed known risk factors such as consumption of RTE dairy, seafood and processed meat and underlined new food vehicles as fruits and vegetables, recently involved in outbreaks. There were not enough data to appraise travel, animal-contact and personto-person as transmission pathways for listeriosis. These results will allow refining the case-control studies in the aim of improving risk factors characterisation for listeriosis in the susceptible population.
\end{abstract}

\section{Introduction}

Listeriosis is a severe foodborne illness, caused by the bacterium Listeria monocytogenes, which is widely distributed in the environment. Listeriosis is a major public health concern as underlined by its hospitalization rate of $98.6 \%$ and a case-fatality ratio of $13.8 \%$ reported in Europe in 2017 (EFSA and ECDC, 2018). The incidence of listeriosis is low, estimated at around 3 to 6 cases per 1 million population per year (de Noordhout et al., 2014). Two clinical forms of listeriosis exist: non-invasive forms mainly with gastroenteritis, often underestimated in several countries by lack of surveillance, and invasive forms with bacteraemia, neurolisteriosis, maternal-neonatal infections and focal infections in various organ systems (Charlier et al., 2017; Ooi and Lorber, 2005). High- risk populations include the elderly ( $>65$ years old), immunocompromised people and pregnant women (EFSA BIOHAZ Panel, 2018). Mainly characterized by sporadic cases or small clusters, listeriosis cases have also occurred as outbreaks and large human clusters identified through epidemiological investigations using whole genome sequencing (WGS) methods (Moura et al., 2017; Nielsen et al., 2017; Van Walle et al., 2018). L. monocytogenes is mainly recognized to be transmitted by the ingestion of ready-to-eat (RTE) foods that are held for extended periods at refrigeration temperatures and allow growth to high numbers at the time of consumption. Investigation of listeriosis outbreaks has identified various food vehicles such as cheeses, RTE meat products, and fish products (Buchanan et al., 2017). New food vehicles, including foods that do not support the growth of $L$. monocytogenes (e.g. ice-cream) have also been identified through recent outbreak investigations (Buchanan et al., 2017).

\footnotetext{
* Corresponding author.

E-mail addresses: alexlec@pasteur.fr (A. Leclercq), Pauline.kooh@anses.fr (P. Kooh), jean-christophe.augustin@vet-alfort.fr (J.-C. Augustin), Laurent.GUILLIER@

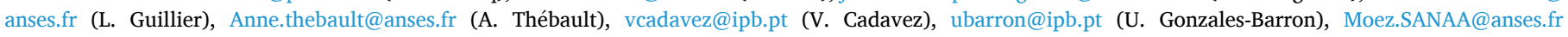
(M. Sanaa).

1 Co-first authors
} 
Case-control studies of sporadic disease are a valuable tool to identify risk factors for human infections, including routes of transmission, food exposures, behavioural and predisposing factors. A systematic review and a meta-analysis of case-control studies were performed in order to combine the association measures, odds-ratios (OR), between listeriosis and its main risk factors.

\section{Material and methods}

The protocol of the systematic review and the meta-analysis model are described in depth in the methodological paper of this special issue (Gonzales-Barron et al., 2019).

\subsection{Systematic review}

The literature search was conducted in March 2017 in five bibliographic search engines, Science Direct, PubMed, Scielo, ISI Web of Science and Scopus. The search strategy was limited to title/ abstract/ keyword using the following keywords: ("Listeria monocytogenes" "OR" "listeriosis") AND ("case-control" OR "risk factor" "OR" "cohort") AND ("infection" $O R$ "disease"). No restrictions were defined for the year of the article or type of publication. The search was limited to the languages English, French, Portuguese and Spanish.

Each reference record was screened for relevance for inclusion in the meta-analysis study. The methodological quality of the "candidate" studies was appraised using pre-set quality criteria comprising (1) appropriate selection of the controls; (2) adjustment to correct for confounders; (3) comparability between cases and controls; (4) acceptable responses rates for the exposed and control groups; (5) data analysis appropriate to the study design; (6) provision of Odd ratio (OR) with confidence interval or $p$-value; or provision of sufficient data to calculate ORs; (7) overall quality of the study (Gonzales-Barron et al., 2019). Primary studies that passed the screening for relevance were marked as having potential for bias if they failed to meet at least one of the methodological quality assessment criteria.

Data from primary studies were then extracted using a standardised spreadsheet. Data extracted included the relevant study characteristics (Country, year, population, serotype/phage type/strains, case definition, design, sample size of the groups, type of model, matching and adjusting criteria), the categorized risk factors, the setting, the handling practices and the outcome of the study (OR).

A data categorization scheme was established to hierarchically group the risk factors into travel, host-specific factors and pathways of exposure (see the methodological paper of this issue (Gonzales-Barron et al., 2019)). Specific partitions were made to investigate the risk related to ready-to-eat foods (i.e., dairy, meat, seafood and produce RTE). The variable "Population" was stratified into specific populations taking into account their respective susceptibility and the clinical form of listeriosis, namely pregnancy related cases ("perinatal") and other forms of invasive listeriosis ("non-perinatal").

\subsection{Data synthesis}

As described in Gonzales-Barron et al. (2019), the joint meta-analytical data was first described using basic statistics. Next, data was partitioned into subsets of categories of risk factors. Meta-analysis models were then fitted to each of the data partitions or subsets in order to estimate the overall OR due to-travel, host specific factors and transmission pathways related to person-to-person contagion, animal contact, environmental exposures and food vehicles. The meta-analytical models were fitted separately by population type. For some food classes, the effects of handling (i.e., eating raw, undercooked) and setting (i.e., eating out) on the overall OR were assessed by the calculation of the ratio of the mean OR when food is mishandled (or, alternatively, when food is prepared outside the home) to the base OR.

The statistical analysis was designed to assess the effect of the

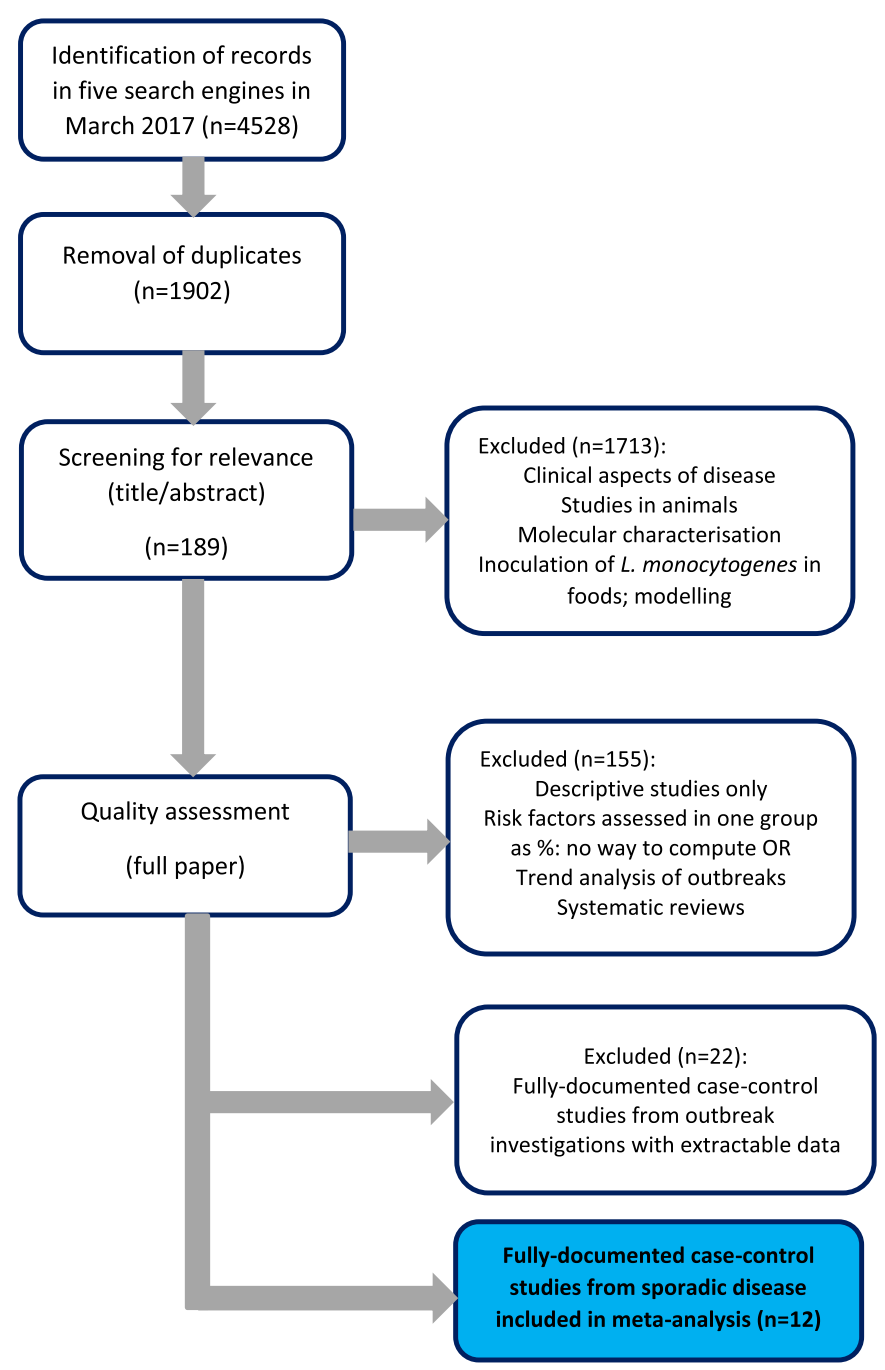

Fig. 1. PRISMA flowchart for the selection of case-control studies of human listeriosis included in this meta-analysis.

geographical region, the study period and the analysis type (univariate/ multivariate) on the final result. The objective of the region-specific meta-analysis was to inform the decision on the geographical regions that should be maintained for the subsequent pooling of OR. All metaanalysis models were essentially weighted random-effects linear regression models. Once a meta-analysis model was fitted, influential diagnostics statistics were applied in order to remove any influential observation originating from studies marked as having potential-forbias. Publication bias was assessed by funnel plots and a statistical test investigating the effect of the study sample size on the ORs (Gonzales-Barron et al., 2019). Heterogeneity between studies was assessed by different indicators, such as the between-study variability $\left(\tau^{2}\right)$, the QE test investigating residual heterogeneity, the variance of residuals and the intra-class correlation $I^{2}$ (Gonzales-Barron et al., 2019). Publication bias and remaining heterogeneity were not further corrected for, but were taken into account for the interpretation of the results.

All analyses were produced in the R software (R Development Core Team, 2008) implemented with the metafor package (Viechtbauer, 2010).

\section{Results}

\subsection{Descriptive statistics}

From 1902 identified references, 189 passed the relevance screening 
Table 1

Characteristics of case-control studies investigating sources of sporadic human listeriosis included in the meta-analysis.

\begin{tabular}{|c|c|c|c|c|c|c|c|}
\hline Study ID* & Country & $\begin{array}{l}\text { Study } \\
\text { period }\end{array}$ & Population & Design & $\begin{array}{l}\text { Analysis \& } \\
\text { model }^{* * *}\end{array}$ & $\begin{array}{l}\text { Cases/ } \\
\text { controls }\end{array}$ & Quality \\
\hline Dalton et al. 2011 & Australia & 2001-2004 & $\begin{array}{l}\text { Non-perinatal } \\
\text { (immuno-compromised) } \\
\text { Perinatal }\end{array}$ & $\begin{array}{l}\text { Matched } \\
\text { Matched }\end{array}$ & $\begin{array}{l}\text { Uni-CL } \\
\text { Multi-CL } \\
\text { Uni-UL } \\
\text { Multi-UL }\end{array}$ & $\begin{array}{l}117 \text { cases } \\
85 \text { controls } \\
19 \text { cases } \\
12 \text { controls }\end{array}$ & Good \\
\hline $\begin{array}{l}\text { Fernández et al. } \\
2009\end{array}$ & Spain & $1995-2007$ & $\begin{array}{l}\text { Non-perinatal } \\
\text { (transplant-recipients) }\end{array}$ & Matched & $\begin{array}{l}\text { Uni-Chi } \\
\text { Multi-CL }\end{array}$ & $\begin{array}{l}30 \text { cases } \\
60 \text { controls }\end{array}$ & Good \\
\hline Friesema et al. 2015 & Netherlands & $2008-2013$ & $\begin{array}{l}\text { Non-perinatal } \\
\text { (immuno-compromised) }\end{array}$ & Unmatched & $\begin{array}{l}\text { Uni-UL } \\
\text { Multi-UL }\end{array}$ & $\begin{array}{l}279 \text { cases } \\
1733 \text { controls }\end{array}$ & Good \\
\hline Gillespie et al. 2010a & UK & 2001-2007 & $\begin{array}{l}\text { Peri/non-peri } \\
\text { Non-perinatal } \\
\text { (elderly) }\end{array}$ & $\begin{array}{l}\text { Unmatched } \\
\text { Unmatched }\end{array}$ & $\begin{array}{l}\text { Uni-Chi } \\
\text { Uni-Chi }\end{array}$ & $\begin{array}{l}171 \text { cases } \\
60646 \\
\text { controls } \\
104 \text { cases } \\
15177 \\
\text { controls }\end{array}$ & Poor \\
\hline $\begin{array}{l}\text { Gillespie et al. } \\
\text { 2010b }\end{array}$ & UK & $2005-2008$ & $\begin{array}{l}\text { Non-perinatal } \\
\text { (Elderly) }\end{array}$ & Unmatched & Uni-Chi & $\begin{array}{l}159 \text { cases } \\
18115 \\
\text { controls }\end{array}$ & Good \\
\hline Jensen et al. 1994 & Denmark & $1989-1990$ & $\begin{array}{l}\text { Perinatal/non-perinatal } \\
\text { (pregnant and immuno- } \\
\text { compromised) }\end{array}$ & Unmatched & Uni-Chi & $\begin{array}{l}66 \text { cases } \\
33 \text { controls }\end{array}$ & Poor \\
\hline Linnan et al. 1988 & USA & $1985-1987$ & Perinatal/non-perinatal & Unmatched & Uni-Chi & $\begin{array}{l}\text { ? cases } \\
\text { ? controls }\end{array}$ & Good \\
\hline Preussel et al. 2015 & Germany & $2012-2013$ & $\begin{array}{l}\text { Non-perinatal } \\
\text { (Immuno-compromised) }\end{array}$ & Unmatched & $\begin{array}{l}\text { Uni-UL } \\
\text { Multi-UL }\end{array}$ & $\begin{array}{l}109 \text { cases } \\
1982 \text { controls }\end{array}$ & Good \\
\hline Schlech et al. 2005 & Canada & $2002-2004$ & $\begin{array}{l}\text { Non-perinatal } \\
\text { (Underlying GI diseases) }\end{array}$ & Unmatched & Uni-Chi & $\begin{array}{l}12 \text { cases } \\
24 \text { cases }\end{array}$ & Poor \\
\hline Schuchat et al. 1992 & USA & $1988-1990$ & $\begin{array}{l}\text { Perinatal/non-perinatal } \\
\text { (pregnant and immuno- } \\
\text { compromised) }\end{array}$ & Matched & $\begin{array}{l}\text { Uni-MH } \\
\text { Multi-CL }\end{array}$ & $\begin{array}{l}165 \text { cases } \\
376 \text { controls }\end{array}$ & Good \\
\hline Schwartz et al. 1988 & USA & $1986-1987$ & $\begin{array}{l}\text { Perinatal/non-perinatal } \\
\text { (pregnant and immuno- } \\
\text { compromised) }\end{array}$ & Matched & $\begin{array}{l}\text { Uni-MH } \\
\text { Multi-CL }\end{array}$ & $\begin{array}{l}80 \text { cases } \\
239 \text { controls }\end{array}$ & Good \\
\hline Varma et al. 2007 & USA & $2000-2003$ & $\begin{array}{l}\text { Perinatal/non-perinatal } \\
\text { (pregnant and immuno- } \\
\text { compromised) }\end{array}$ & $\begin{array}{l}\text { Unmatched (frequency- } \\
\text { matched) }\end{array}$ & $\begin{array}{l}\text { Uni-UL } \\
\text { Multi-UL }\end{array}$ & $\begin{array}{l}169 \text { cases } \\
376 \text { controls }\end{array}$ & Good \\
\hline
\end{tabular}

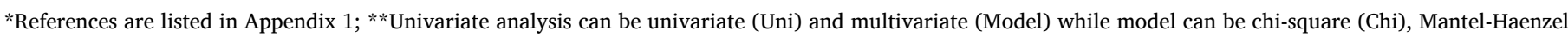
(MH), unconditional logistic (UL) and conditional logistic (CL).

and 12 passed the quality assessment stage (Fig. 1). Table 1 summarizes the main features of the case-control studies used in this meta-analysis. These 12 primary studies investigating sporadic listeriosis were conducted between 1985 and 2013 and provided 226 ORs. A total of $84 \%$ of the meta-analytical data were produced by case-control studies from Australia (71 ORs), USA (56 ORs), Germany ( 35 ORs) and UK (27 ORs).

All studies targeted susceptible populations. Seven case-control studies investigated exposures in the non-perinatal population comprising immunocompromised and elderly (139 ORs), six casecontrol studies focused on the broad susceptible population with no distinction between perinatal and non-perinatal cases (labelled as "perinatal/non-perinatal" - 63 ORs) and one study (Dalton et al., 2011) conducted a case-control investigation on the perinatal population (24 ORs extracted). Because the amount of data for the perinatal population was limited, separate meta-analysis models could not be adjusted for this population class. However, separate meta-analyses could be fitted for the non-perinatal population (139 ORs) and to the broad susceptible population (combined non-perinatal, perinatal and perinatal/non-perinatal data of $226 \mathrm{ORs}$ ).

The majority of primary studies investigated listeriosis caused by undifferentiated serotypes, except for Varma et al. (2007) whose case patients were infected with either serotype $1 / 2 \mathrm{a}$ or $4 \mathrm{~b}$. In all studies, the cases of listeriosis were laboratory-confirmed.

With regards to the risk factor classes, sporadic illness investigations focused on host specific factors (68 OR) and multiple pathways of exposure: food (149 ORs), environment ( 8 ORs) and contact with animals (1 OR). Travel and person-to-person contagion were not investigated as potential risk factors for listeriosis among the case-control studies included in this meta-analysis.
After methodological quality assessment, three case-control studies were marked as being below standards. In Gillespie et al. (2010), controls were not necessarily healthy people, whilst in Schlech et al. (2005), controls were patients with campylobacteriosis or salmonellosis. Finally, the OR measures from Jensen et al. (1994) were assigned the potential-for-bias status because the study, in general terms, was not clearly described, and some of the OR extracted were approximated. Those three case-control studies furnished 16 potentially biased OR whose influence on the pooled OR estimates was appraised by means of the Cook's distance. Whenever they were determined to be influential, they were removed from the meta-analysis models (Tables 2, 3, 4, 5).

Four case-control studies employed a matched experimental design and produced a total of 103 matched ORs. Bringing together the matched and unmatched designs, 127 extracted OR were not adjusted by any confounder (crude OR), while 100 OR were adjusted using either Mantel-Haenzel method or logistic regressions.

\subsection{Meta-analysis}

For every data partition, the meta-analysed risk factors are presented in summary tables only when significant (Tables 2, 3, 4). Pooled ORs were considered as significant when the lower bound of the $95 \%$ CI was equal or greater than 1 . Non-significant results on main risk factors are presented in Supplementary Material 2. More detailed descriptive results, in particular funnel plots, forest plots, and OR of non-significant results, are in a complete report available upon request.

The pathways of exposure that on meta-analysis had non-significant association with listeriosis were farm environment and food subcategories such as vegetables, red meats, crustaceans, molluscs, 
Table 2

Results of the meta-analysis on the main risk factors.

\begin{tabular}{|c|c|c|c|c|c|c|c|}
\hline Population & Risk factor & Pooled OR $[95 \% \mathrm{CI}]$ & $\begin{array}{l}\mathrm{N} / \\
\mathrm{n}^{*}\end{array}$ & $p$-value of risk factor & $\begin{array}{l}\text { Publication } \\
\text { bias } p \text {-value }\end{array}$ & $\begin{array}{l}\text { Points } \\
\text { removed ** }\end{array}$ & $\begin{array}{l}\text { Heterogeneity } \\
\text { analysis*** }\end{array}$ \\
\hline \multicolumn{8}{|c|}{ Host-specific } \\
\hline \multirow{6}{*}{ All susceptible } & Other medical conditions & $3.020[2.326-3.923]$ & $8 / 24$ & $<0.0001$ & \multirow{6}{*}{$<0.0001$} & \multirow{6}{*}{0} & $\tau^{2}=2.015 \mathrm{QE}(\mathrm{df}$ \\
\hline & Antiacids & $2.014[1.260-3.218]$ & $5 / 9$ & 0.003 & & & $=61)=$ \\
\hline & Immunocompromising conditions & $5.170[1.735-15.407]$ & $5 / 21$ & 0.003 & & & 437.088, p-val $<$ \\
\hline & Chronic diseases & $2.927[1.913-4.480]$ & $3 / 11$ & $<0.0001$ & & & 0.0001 \\
\hline & & & & & & & $S^{2}=1.180$ \\
\hline & & & & & & & $\mathrm{I}^{2}=63.06$ \\
\hline \multirow{6}{*}{ All susceptible } & & & ood & & & & \\
\hline & Produce & $1.415[1.003-1.995]$ & $7 / 27$ & 0.048 & \multirow{6}{*}{0.001} & \multirow{6}{*}{1} & $\tau^{2}=0.688 \mathrm{QE}(\mathrm{df}$ \\
\hline & Meat & $1.371[1.027-1.830]$ & $8 / 44$ & 0.032 & & & $=136)=$ \\
\hline & Dairy & $1.867[1.292-2.699]$ & $9 / 45$ & 0.001 & & & 615.478, p-val < \\
\hline & Seafood & $2.148[1.190-3.877]$ & $4 / 14$ & 0.011 & & & 0.0001 \\
\hline & Composite & $1.621[1.014-2.590]$ & $4 / 11$ & 0.044 & & & $S^{2}=0.549$ \\
\hline & & & & & & & $\mathrm{I}^{2}=55.61$ \\
\hline \multirow{5}{*}{ Non-perinatal } & Dairy & $1.605[1.187-2.170]$ & $4 / 27$ & 0.002 & \multirow{5}{*}{0.807} & \multirow{5}{*}{1} & $\tau^{2}=1.087 \mathrm{QE}(\mathrm{df}$ \\
\hline & Seafood & 2.477 [1.098 - 5.59] & $3 / 9$ & 0.029 & & & $=69)=$ \\
\hline & & & & & & & 439.237, p-val \\
\hline & & & & & & & $<0.0001$ \\
\hline & & & & & & & $\begin{array}{l}\mathrm{S}=0.510 \\
\mathrm{I}^{2}=67.80\end{array}$ \\
\hline
\end{tabular}

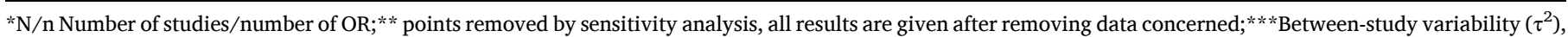
test for residual heterogeneity $(\mathrm{QE})$, variance of residuals $\left(\mathrm{s}^{2}\right)$, intra-class correlation $\left(\mathrm{I}^{2}\right)$.

Table 3

Results of the meta-analysis on ready-to-eat foods.

\begin{tabular}{|c|c|c|c|c|c|c|c|}
\hline Population & $\begin{array}{l}\text { Type of RTE } \\
\text { food }\end{array}$ & $\begin{array}{l}\text { Pooled OR [95\% } \\
\text { CI] }\end{array}$ & $\begin{array}{l}\mathrm{N} / \\
\mathrm{n}^{*}\end{array}$ & $\begin{array}{l}\mathrm{p} \text {-value of risk } \\
\text { factor }\end{array}$ & $\begin{array}{l}\text { Publication bias } p \text { - } \\
\text { value }\end{array}$ & $\begin{array}{l}\text { Points removed } \\
* *\end{array}$ & Heterogeneity analysis*** \\
\hline \multirow{3}{*}{$\begin{array}{l}\text { All } \\
\text { susceptible }\end{array}$} & Dairy & $1.830[1.252-$ & $8 /$ & 0.002 & \multirow{3}{*}{0.553} & \multirow{3}{*}{0} & \multirow{3}{*}{$\begin{array}{l}\tau^{2}=3.177 \mathrm{QE}(\mathrm{df}=77)=410.8, \text { p-val }<0.0001 \\
\mathrm{~S}^{2}=0.510 \mathrm{I}^{2}=86.16\end{array}$} \\
\hline & & $2.676]$ & 44 & & & & \\
\hline & Seafood & $\begin{array}{l}6.273[1.457- \\
27.01]\end{array}$ & $3 / 6$ & 0.014 & & & \\
\hline \multirow{3}{*}{$\begin{array}{l}\text { Non } \\
\text { perinatal }\end{array}$} & Dairy & $1.636[1.189-$ & 4/ & 0.003 & \multirow{3}{*}{0.610} & \multirow{3}{*}{0} & \multirow{3}{*}{$\begin{array}{l}\tau^{2}=2964 \mathrm{QE}(\mathrm{df}=48)=291.1, \mathrm{p}-\mathrm{val}<0.0001 \\
\mathrm{~S}^{2}=0.608 \mathrm{I}^{2}=83.00\end{array}$} \\
\hline & & $2.250]$ & 27 & & & & \\
\hline & Seafood & $\begin{array}{l}10.746[1.541- \\
74.91]\end{array}$ & $2 / 4$ & 0.017 & & & \\
\hline
\end{tabular}

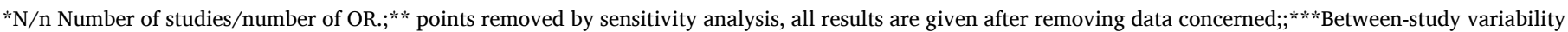
$\left(\tau^{2}\right)$, test for residual heterogeneity (QE), variance of residuals $\left(\mathrm{s}^{2}\right)$, intra-class correlation $\left(\mathrm{I}^{2}\right)$.

Table 4

Results of the meta-analysis on disaggregated risk factors.

\begin{tabular}{|c|c|c|c|c|c|c|c|c|}
\hline Risk factor & Population & $\begin{array}{l}\text { Risk factor } \\
\text { precise }\end{array}$ & $\begin{array}{l}\text { Pooled OR } \\
{[95 \% \mathrm{CI}]}\end{array}$ & $\begin{array}{l}\mathrm{N} / \\
\mathrm{n}^{*}\end{array}$ & $\begin{array}{l}p \text {-value of risk } \\
\text { factor }\end{array}$ & $\begin{array}{l}\text { Publication bias } \\
p \text {-value }\end{array}$ & $\begin{array}{l}\text { Points } \\
\text { removed } * *\end{array}$ & Heterogeneity analysis*** \\
\hline Meat & $\begin{array}{l}\text { All } \\
\text { susceptible }\end{array}$ & $\begin{array}{l}\text { Poultry } \\
\text { Processed } \\
\text { meat }\end{array}$ & $\begin{array}{l}2.157[1.177- \\
3.951] \\
1.624[1.230- \\
2.143]\end{array}$ & $\begin{array}{l}6 / \\
13\end{array}$ & 0.013 & 0.002 & 0 & $\begin{array}{l}\tau^{2}=0.174 \mathrm{QE}(\mathrm{df}=22)=89.90, \mathrm{p}-\mathrm{val}< \\
0.0001 \mathrm{~S}^{2}=0.625 \mathrm{I}^{2}=21.75\end{array}$ \\
\hline Meat & $\begin{array}{l}\text { Non } \\
\text { perinatal }\end{array}$ & $\begin{array}{l}\text { Processed } \\
\text { meat }\end{array}$ & $\begin{array}{l}1.549[1.307- \\
1.836]\end{array}$ & $3 / 7$ & $<0.0001$ & 0.226 & 0 & $\begin{array}{l}\tau^{2}=0.092 \mathrm{QE}(\mathrm{df}=11)=63.06, \mathrm{p}-\mathrm{val} \\
<0.0001 \mathrm{~S}^{2}=0.277 \mathrm{I}^{2}=24.92\end{array}$ \\
\hline Dairy & $\begin{array}{l}\text { All } \\
\text { susceptible }\end{array}$ & Cheese & $\begin{array}{l}1.832[1.270- \\
2.643] \\
2.139[1.314- \\
3.481]\end{array}$ & $\begin{array}{l}8 / \\
40 \\
3 / 5\end{array}$ & 0.001 & 0.813 & 0 & $\begin{array}{l}\tau^{2}=0.305 \mathrm{QE}(\mathrm{df}=43)=196.182, \mathrm{p}-\mathrm{val} \\
<0.0001 \mathrm{~S}^{2}=0.417 \mathrm{I}^{2}=42.21\end{array}$ \\
\hline Dairy & $\begin{array}{l}\text { Non } \\
\text { perinatal }\end{array}$ & Cheese & $\begin{array}{l}1.586[1.188- \\
2.119]\end{array}$ & $\begin{array}{l}4 / \\
24\end{array}$ & 0.002 & 0.160 & 0 & $\begin{array}{l}\tau^{2}=0.000 \mathrm{QE}(\mathrm{df}=25)=141.29, \mathrm{p} \text {-val } \\
<0.0001 \mathrm{~S}^{2}=0.396 \mathrm{I}^{2}=0.00\end{array}$ \\
\hline Produce & $\begin{array}{l}\text { All } \\
\text { susceptible }\end{array}$ & Fruits & $\begin{array}{l}1.538[1.1431- \\
2.070]\end{array}$ & $\begin{array}{l}2 / \\
11\end{array}$ & 0.005 & 0.002 & 0 & $\begin{array}{l}\tau^{2}=0.054 \mathrm{QE}(\mathrm{df}=25)=53.736, \mathrm{p}-\mathrm{val}= \\
0.001 \mathrm{~S}^{2}=0.209 \mathrm{I}^{2}=20.67\end{array}$ \\
\hline Composite & $\begin{array}{l}\text { All } \\
\text { susceptible }\end{array}$ & RTE & $\begin{array}{l}1.486[1.1263- \\
1.960]\end{array}$ & $2 / 3$ & 0.005 & $<0.0001$ & 0 & $\begin{array}{l}\tau^{2}=0.244 \mathrm{QE}(\mathrm{df}=9)=24.60, p-\mathrm{val}= \\
0.003 \mathrm{~S}^{2}=0.397 \mathrm{I}^{2}=38.07\end{array}$ \\
\hline
\end{tabular}

*N/n Number of studies/number of OR.;*** points removed by sensitivity analysis, all results are given after removing data concerned;; ;***Between-study variability $\left(\tau^{2}\right)$, test for residual heterogeneity $(\mathrm{QE})$, variance of residuals $\left(\mathrm{s}^{2}\right)$, intra-class correlation $\left(\mathrm{I}^{2}\right)$.

processed seafood and composite dishes (Supplementary Material 2).

\subsection{Meta-analysis for host specific risk factors}

The meta-analysis on host-specific factors showed that, immunocompromising conditions, other medical conditions, chronic diseases and the use of anti-acids exacerbated the risk of acquiring listeriosis in all of the geographical regions with pooled OR between 2.014 and 5.170. Suffering from any immunocompromising condition (pooled OR=5.170; 95\% CI [1.735 - 15.407]), was the most important 
Table 5

Effect of food handling on the pooled OR.

\begin{tabular}{|c|c|c|c|c|c|c|c|c|}
\hline Risk factor & $\begin{array}{l}\text { Risk factor } \\
\text { precise }\end{array}$ & $\begin{array}{l}\text { Pooled OR } \\
{[95 \% \mathrm{CI}]}\end{array}$ & $\begin{array}{l}\mathrm{N} / \\
\mathrm{n}^{*}\end{array}$ & $\begin{array}{l}p \text {-value of } \\
\text { risk factor }\end{array}$ & $\begin{array}{l}\text { Increase in OR due to } \\
\text { poor handling [ } 95 \% \\
\text { CI] }\end{array}$ & $\begin{array}{l}\text { Points } \\
\text { removed } * *\end{array}$ & $\begin{array}{l}\text { Publication bias } \\
p \text {-value }\end{array}$ & Heterogeneity analysis*** \\
\hline \multirow{4}{*}{$\begin{array}{l}\text { Processed meat } \\
\text { and poultry (at) }\end{array}$} & Undercooked & $5.013[1.776$ & $3 /$ & 0.001 & 2.168 [1.297 - 3.623] & \multirow{4}{*}{0} & \multirow{4}{*}{0.069} & \multirow{4}{*}{$\begin{array}{l}\tau^{2}=0.052 \mathrm{QE}(\mathrm{df}=30)=63.10, \mathrm{p}- \\
\mathrm{val}=0.001 \mathrm{~S}^{2}=0.487 \mathrm{I}^{2}=9.681\end{array}$} \\
\hline & \multirow{3}{*}{ Base } & $-14.142]$ & 7 & \multirow{3}{*}{0.003} & \multirow{3}{*}{ J } & & & \\
\hline & & $2.312[1.370$ & 7/ & & & & & \\
\hline & & $-3.904]$ & 26 & & & & & \\
\hline \multirow{3}{*}{ Fruits } & Eating out & $2.506[1.216$ & $1 /$ & 0.001 & $2.358[1.483-3.750]$ & \multirow{3}{*}{0} & \multirow{3}{*}{0.077} & \multirow{3}{*}{$\begin{array}{l}\tau^{2}=0.000 \mathrm{QE}(\mathrm{df}=9)=5.945, \mathrm{p} \\
\mathrm{val}=0.745 \mathrm{~S}^{2}=0.226 \mathrm{I}^{2}=0.000\end{array}$} \\
\hline & & - 5.167] & 3 & & & & & \\
\hline & Base & $\begin{array}{l}1.063[0.820 \\
-1.378]\end{array}$ & $\begin{array}{l}2 / \\
8\end{array}$ & 0.645 & - & & & \\
\hline
\end{tabular}

*N/n Number of studies/number of OR; ** points removed by sensitivity analysis, all results are given after removing data concerned;***Between-study variability $\left(\tau^{2}\right)$, test for residual heterogeneity $(\mathrm{QE})$, variance of residuals $\left(\mathrm{s}^{2}\right)$, intra-class correlation $\left(\mathrm{I}^{2}\right)$.

\begin{tabular}{|c|c|c|c|c|c|}
\hline Study & Country & Label & Odds Ratio & {$[95 \% \mathrm{Cl}]$} & \\
\hline Dalton_Epilnf_2011 & Australia & Chemotherapy & 1.7 & {$[0.6-5]$} & $\longrightarrow$ \\
\hline Dalton_Epilnf_2011 & Australia & Radiation theraphy & 0.8 & {$[0.1-4.4] \longrightarrow$} & \\
\hline Friesema_Eurosurveillance_2015* & Netherlands & Immune disorder & 14.7 & {$[7.4-29.1]$} & $\longrightarrow$ \\
\hline Friesema_Eurosurveillance_2015* & Netherlands & Cancer & 24.8 & [15.4-40] & $\rightarrow$ \\
\hline Friesema_Eurosurveillance_2015* & Netherlands & Organ transplant & 138.9 & [15.7-Inf] & $\rightarrow$ \\
\hline Friesema_Eurosurveillance_2015* & Netherlands & Use of immunosuppressants & 80.3 & {$[49.8-129.7]$} & \\
\hline Friesema_Eurosurveillance_2015* & Netherlands & Immune disorder & 3.3 & [1.3-8.6] & 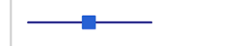 \\
\hline Friesema_Eurosurveillance_2015* & Netherlands & Cancer & 26.8 & {$[14.4-49.8]$} & $\longrightarrow$ \\
\hline Friesema_Eurosurveillance_2015* & Netherlands & Use of immunosuppressants & 53.7 & {$[31-93]$} & $\rightarrow$ \\
\hline PreuBel_PLOSONE_2015* & Germany & Chemotherapy / 3 mo & 17.12 & {$[8.17-35.78]$} & $\longrightarrow$ \\
\hline PreuBel_PLOSONE_2015* & Germany & Immunosupressive med /3 mo & 6.52 & {$[4.23-10.04]$} & \\
\hline PreuBel_PLOSONE_2015* & Germany & Radiation therapy /3 mo & 5.77 & {$[2.08-16.03]$} & \\
\hline PreuBel_PLOSONE_2015* & Germany & Hemodialysis $/ 3$ mo & 5.44 & {$[1.75-16.96]$} & - \\
\hline PreuBel_PLOSONE_2015* & Germany & Cancer-hematological /5 yrs & 15.97 & {$[6.51-39.16]$} & $\longrightarrow$ \\
\hline PreuBel_PLOSONE_2015* & Germany & Solid organ transplantation & 3.89 & {$[1.3-11.62]$} & \\
\hline PreuBel_PLOSONE_2015* & Germany & Autoimmune disorder & 3.53 & {$[1.68-7.41]$} & \\
\hline PreuBel_PLOSONE_2015* & Germany & Cancer $/ 5$ yrs & 1.63 & {$[0.93-2.84]$} & $\longrightarrow$ \\
\hline PreuBel_PLOSONE_2015* & Germany & Immunosupressive med /3 mo & 8.75 & {$[4.91-15.58]$} & \\
\hline PreuBel_PLOSONE_2015* & Germany & Immunocompromising disease & 2.73 & {$[1.44-5.2]$} & \\
\hline FernandezSabe_CID_2009 & Spain & Receipt of prior transplant & 1.22 & {$[0.27-5.49]$} & \\
\hline Jensen_SJID_1994 & Denmark & Steroids \& chemotherapy treatment & 30.5 & {$[4.3-217]$} & \\
\hline \multirow[t]{2}{*}{ Random Effect Meta-Analysis } & All & & 5.17 & {$[1.74-15.41]$} & \\
\hline & & & & $\begin{array}{c} \\
0.10\end{array}$ & \begin{tabular}{l|l|l} 
& 1 & \\
5.0 & 10.0 & 25.0
\end{tabular} \\
\hline
\end{tabular}

Fig. 2. Forest-plot of the association of listeriosis with immunocompromising conditions in the general susceptible population ( $\mathrm{n}=21)\left({ }^{*}\right.$ adjusted OR)

predisposing factor for listeriosis among the susceptible population. Immunocompromising conditions included cancer, chemotherapy, and transplant (Fig. 2). Other medical conditions (pooled OR=3.020; 95\% CI [2.326 - 3.923]) included cardiovascular diseases, pre-existing liver disease, previous hospitalization, gastrointestinal diseases, and lung diseases.

\subsection{Meta-analysis for food consumption}

Most of the routes of transmission of listeriosis recovered in the systematic review were related to consumption of foods such as meat, dairy, seafood, composite dishes and produce. Very limited data were available for food subcategories such as eggs, grains and beverages (juice), so the significance of these sources as potential vehicles of transmission of listeriosis could not be appraised.

The food categories followed a similar ranking as sources of listeriosis in the general susceptible population and the non-perinatal subset. According to their association with listeriosis in the susceptible population, the global food categories ranked in decreasing order were: seafood (pooled $\mathrm{OR}=2.148$; 95\% CI [1.190 - 3.877)), dairy (pooled $\mathrm{OR}=1.867 ; 95 \%$ CI $[1.292-2.699]$ ), composite foods (pooled $\mathrm{OR}=1.621 ; 95 \% \mathrm{CI}[1.014-2.590]$ ), produce (pooled $\mathrm{OR}=1.41595 \% \mathrm{CI}$ $[1.003-1.995])$ and meat (pooled OR $=1.371[1.027-1.830]$ ) (Table 2).

The meta-analyses by RTE class revealed that the consumption of RTE seafood (pooled OR ranged from 6.273 to 10.746) and dairy products (pooled OR ranged from 1.636 to 1.830) are the main risk factors for listeriosis in the general susceptible population and the nonperinatal population subset (Table 3 ).

The meta-analysis on the seafood data partition did not reveal significant associations for crustaceans (pooled OR $=1.033$; 95\% CI $[0.677$ - 1.574]), molluscs (pooled OR=1.985; 95\% CI [0.984 - 4.004]) and 


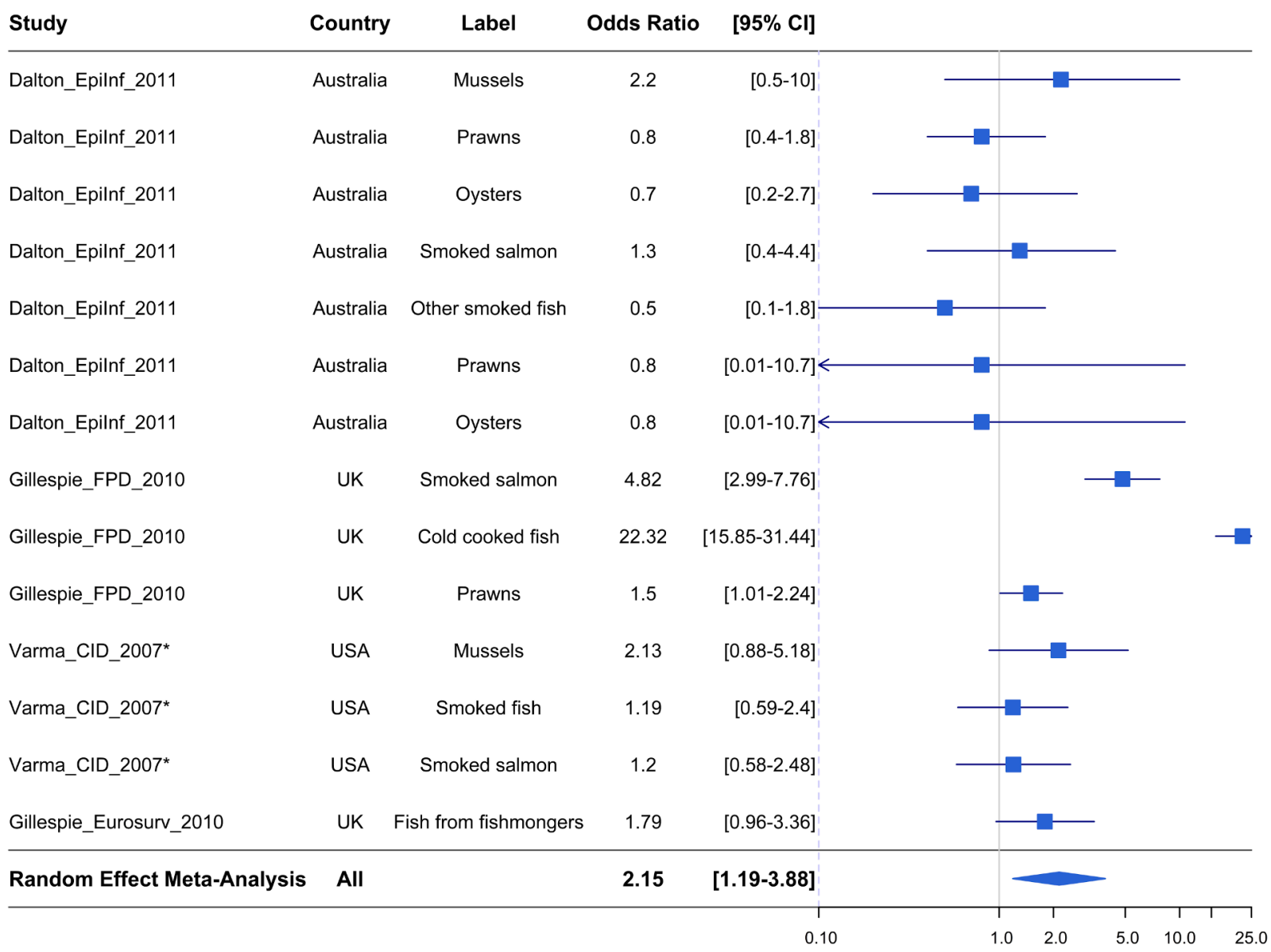

Fig. 3. Forest-plot of the association of listeriosis with the consumption of seafood in the general susceptible population ( $\mathrm{n}=14$ ) (*adjusted OR) .

processed fish (pooled OR=2.790; 95\% CI [0.981 - 7.932]) (cf. Supplementary Material 2).

Within dairy, the consumption of cheese (in majority soft cheese; pooled $\mathrm{OR}=1.832 ; 95 \%$ CI $[1.270-2.643]$ ) and fats (raw milk, raw cream and raw butter; pooled $\mathrm{OR}=2.139 ; 95 \%$ CI [1.314 - 3.481]) were significantly associated with listeriosis in the general susceptible population (Table 4; Fig. 4).

Within meats, the higher association with disease in the susceptible population was found for poultry (essentially undercooked poultry; pooled OR=2.157; 95\% CI [1.177 - 3.951]), although it should be kept in mind that only 5 ORs were available for this pathway of exposure. Moreover, a significant association with listeriosis was found for the consumption of processed meats (pooled OR $=1.624$; 95\% CI $[1.230$ 2.143]) that included processed pork, processed poultry, cooked sausages, raw fermented spreadable sausages, dry-cured ham, deli meats, hotdogs, pate, cold meats and uncooked hotdogs.

Within produce, a significant association was found for the consumption of fruits (melons, cantaloupe, strawberries, RTE fruit salads; pooled $\mathrm{OR}=1.538 ; 95 \%$ CI $[1.1431-2.070])$ by the susceptible population.

\subsection{Meta-analysis on the effects of handling and preparation of foods}

For some food classes, for which relevant information was available, the effects of handling (raw and undercooked) and setting (eating out) were appraised (Table 5). The data partitions suitable for this analysis were: (i) processed meats and poultry, and (ii) fruits.

On meta-analysis, it was found that susceptible people who claimed having eaten raw processed meats or undercooked poultry had their odds of infection significantly increased by a factor of 2.168 . Eating out came up as a significant factor increasing the risk of listeriosis infection. On average, susceptible people who had consumed fruits prepared in a food establishment had theirs their odds of infection significantly increased by a factor of 2.358 .

For some partitions (whole food, meat, produce, composite), both the formal tests and the funnel plots indicated that publication bias is likely (Fig. 5). A significant publication bias $p$-value implies that the OR value measured by the researchers depends upon the sample size. In this case, it is likely that small-sized studies have remained unpublished because of their failure to detect significant OR (Gonzales-Barron et al., 2019). Moreover, the intra-class correlation $I^{2}$ indicates low to moderate heterogeneity $(<75 \%)$ for most of the data partitions (Tables $2,3,4,5)$.

\section{Discussion}

The meta-analysis showed that underlying health conditions or diseases and the consumption of RTE foods (seafood, dairy and meat) were the most important risk factors for sporadic listeriosis. Host-specific related risk factors presented higher pooled OR ranging from 2.014 to 5.170 for the susceptible population. These host susceptibility risk factors were also confirmed by analysis of epidemiological data (Pouillot et al., 2015) and the recent prospective cohort study (Charlier et al., 2017). The relative host susceptibility derived for 11 population subgroups, showed that the highest susceptible population (hematological cancer) is a thousand more susceptible than general healthy population (Pouillot et al., 2015).

The pooled OR assigned to main food categories ranged from 1.371 to 2.477. For specific foods such cheeses or processed meat, the odds ratios increase. The highest pooled OR is observed for RTE fish products in the susceptible populations other than pregnant women with a value 


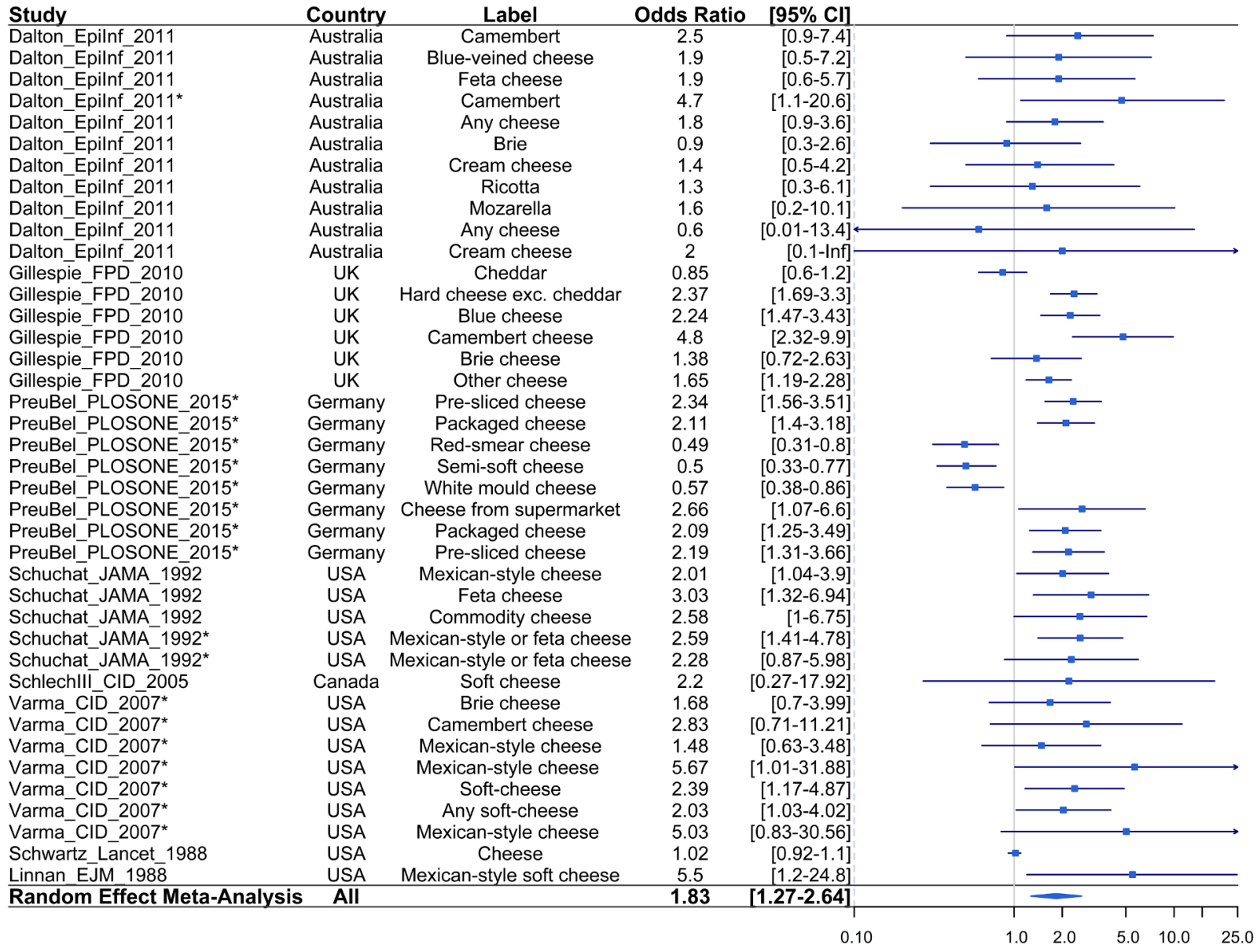

Fig. 4. Forest-plot of the association of listeriosis with consumption of cheeses in the general susceptible population ( $\mathrm{n}=40$ ) (*adjusted OR).

of 10.746 . This probably illustrates the fact that many foods can be contaminated by $L$. monocytogenes and that the practices associated with these foods (manufacturing, storage, consumption) strongly affect the risk associated to them. In these conditions, it is very difficult to identify specific food at risk as the food categories are disparate in terms of ability to support the growth of $L$. monocytogenes. Moreover, the diversity of food questionnaire at national and regional levels concerning the list of foods at risk, the methodology used to perform them and the understanding of patients have always been recognised as a source of bias.

However, this meta-analysis identified the RTE such as seafood, dairy and processed meats as the main risks factors of listeriosis. These food categories have been already identified by more than 100 outbreaks worldwide as foods at risk for listeriosis (Buchanan et al., 2017). The estimated ORs from this meta-analysis are not a direct assessment of attribution of the different food to the sporadic cases. Meanwhile, existing source attribution models published on L. monocytogenes revealed the importance of the same food categories. The EFSA BIOHAZ panel based on a bottom-up approach with data on prevalence, levels of contamination, growth and consumption data together with dose-response assessment yielded estimates that RTE meat products accounted for $67 \%$ of human cases, RTE fish products for $32 \%$ and soft and semi-soft cheeses for 1\% (EFSA BIOHAZ Panel, 2018). Another approach using WGS showed that different source attribution models applied on a set of European human sporadic strains for different levels of molecular analysis (from MLST to wgMLST) tended to place bovine, and thus cheese, as the main source of human listeriosis (32\% to 64\%) (Nielsen et al., 2017). This difference of the importance of cheese could be explained by one assumption done in quantitative risk assessments. Usually, the variability of strain virulence is considered to be the same whatever the food type. However, between-strain virulence variability is huge for L. monocytogenes (Maury et al., 2019). A small change in proportion of most virulent strains in a food category could thus considerably change the estimated contribution of that food.

On the other hand, crustaceans and molluscs are not identified in this meta-analysis as significant factors. In France, crustaceans such as shrimps involved in food safety incidents (withdraw/recall) are contaminated by hypovirulent clones of $L$. monocytogenes such as CC121 and CC9 (Maury et al., 2019, 2016) that could explain the low number of sporadic cases associated to these foods (EFSA and ECDC, 2018; Fritsch et al., 2018; Painset et al., 2019).

Two studies in USA and Australia identified the fruits consumption (cantaloupes) as a risk factor (Dalton et al., 2011; Varma et al., 2007). Cantaloupes present neutral $\mathrm{pH}$ value and thus support the growth of L. monocytogenes (Bassett and McClure, 2008; Hoelzer et al., 2012). More generally, the importance of produce is supported with recent advances in detection of unknown source of listeriosis outbreaks allowed by genomic methods (Buchanan et al., 2017; Chen et al., 2016; EFSA and ECDC, 2018; Nyarko et al., 2016). This point emphasizes the need to develop a risk assessment on produce and especially fruits for L. monocytogenes.

The results of this meta-analysis based on studies conducted before 2013 did not take into account new discovered food vehicles identified thanks to the combination of listeriosis surveillance data and genomic data (Desai et al., 2019). Furthermore, the high discrimination power of genomic methods has recently conducted to the evolution of the definition of sporadic and outbreak cases of listeriosis, distinguishing them more finely (Moura et al., 2017; Van Walle et al., 2018). Food questionnaires shall be permanently updated in each country based on the evolution of food habits and the discovering of new contaminated 

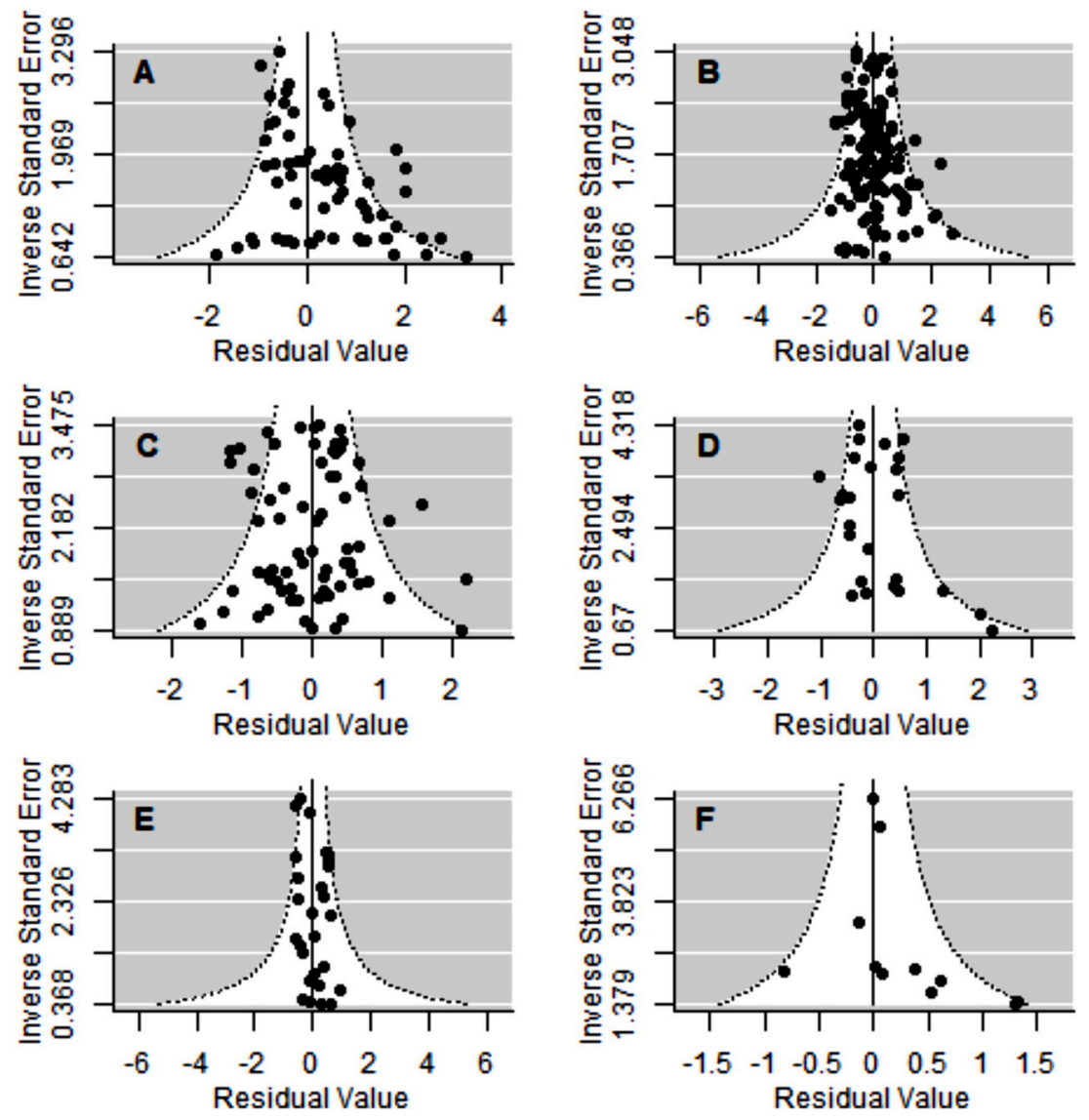

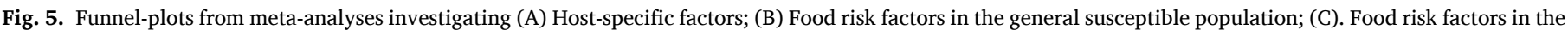

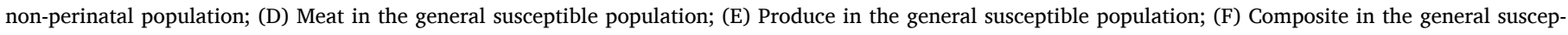
tible population.

products based on food surveillance (EFSA and ECDC, 2018; Self, 2016). Results from the upcoming case control studies might be different because of the change of both the knowledge of the potential food vehicle, the definition of sporadic cases and the new consumption patterns.

The different case-control studies of this meta-analysis published before March 2017 are stored in a database. It will be updated with relevant studies published after this date (e.g. Kvistholm Jensen et al. (2017)) and future case-control studies. Future analysis will help to identify the potential evolution of risk factors.

\section{Conclusions}

This meta-analysis confirmed known risk factors of listeriosis: consumption of RTE food such as milk products or fish products and consumption of processed meat. The risk is probably linked to their intrinsic characteristics allowing the growth of $L$. monocytogenes and their mode of consumption. These risk factors concerned sporadic cases but have also been reported for outbreaks worldwide. A risk assessment related to L. monocytogenes in fruits and vegetables should be investigated based on the increasing consumption of this type of products.

Future case control studies should be conducted by refining the categories of RTE food and including vegetables and fruits that have been the source of human cases. It would be necessary to consider a typology of foods that is more representative of the level of risk and takes into account the processing method (raw, cooked, fermented, etc.), the intrinsic characteristics $(\mathrm{pH}$, water activity, preservatives, background microflora), the storage (short or long shelf-life) and the mode of consumption (immediate consumption, reheating, cooking). It would be interesting to carry out these studies on elderly people that constituted the main part of the susceptible population.

\section{CRediT authorship contribution statement}

Alexandre Leclercq: Writing - original draft. Pauline Kooh: Methodology, Project administration, Writing - original draft. JeanChristophe Augustin: Writing - review \& editing. Laurent Guillier: Writing - review \& editing. Anne Thébault: Methodology, Formal analysis, Visualization. Vasco Cadavez: Methodology, Investigation, Formal analysis. Ursula Gonzales-Barron: Methodology, Investigation, Formal analysis, Writing - review \& editing. Moez Sanaa: Conceptualization, Supervision, Writing - review \& editing.

\section{Declarations of Competing Interest}

The authors declare no conflict of interest.

\section{Acknowledgments}

The authors would like to thank Anses staff and the members of the Anses Working Group on Source Attribution of Foodborne Diseases: Laurence Watier, Frédéric Carlin, Julie David, Philippe Fravalo, Nathalie Jourdan-Da Silva, Lapo Mughini-Gras, Nicole Pavio, Isabelle Villena. U. Gonzales-Barron and V. Cadavez are grateful to the Foundation for Science and Technology (FCT, Portugal) for financial support through national funds FCT/MCTES to CIMO (UIDB/00690/2020). U. GonzalesBarron also thanks FCT, P.I., for the institutional scientific employment program. 


\section{Supplementary materials}

Supplementary material associated with this article can be found, in the online version, at doi:10.1016/j.mran.2020.100128.

\section{References}

Bassett, J., McClure, P., 2008. A risk assessment approach for fresh fruits. J. Appl. Microbiol. 104, 925-943.

Buchanan, R.L., Gorris, L.G.M., Hayman, M.M., Jackson, T.C., Whiting, R.C., 2017. A review of Listeria monocytogenes: an update on outbreaks, virulence, doseresponse, ecology, and risk assessments. Food Control 75, 1-13.

Charlier, C., Perrodeau, E., Leclercq, A., Cazenave, B., Pilmis, B., Henry, B., Lopes, A., Maury, M.M., Moura, A., Goffinet, F., Dieye, H.B., Thouvenot, P., Ungeheuer, M.N., Tourdjman, M., Goulet, V., de Valk, H., Lortholary, O., Ravaud, P., Lecuit, M., group, M.s., 2017. Clinical features and prognostic factors of listeriosis: the MONALISA national prospective cohort study. Lancet Infect. Dis. 17, 510-519.

Chen, Y., Burall, L.S., Luo, Y., Timme, R., Melka, D., Muruvanda, T., Payne, J., Wang, C., Kastanis, G., Maounounen-Laasri, A., De Jesus, A.J., Curry, P.E., Stones, R., K'Aluoch, O., Liu, E., Salter, M., Hammack, T.S., Evans, P.S., Parish, M., Allard, M. W., Datta, A., Strain, E.A., Brown, E.W., 2016. Listeria monocytogenes in stone fruits linked to a multistate outbreak: enumeration of cells and whole-genome sequencing. Appl. Environ. Microbiol. 82, 7030-7040.

Dalton, C.B., Merritt, T.D., Unicomb, L.E., Kirk, M.D., Stafford, R.J., Lalor, K., OzFoodNet Working, G., 2011. A national case-control study of risk factors for listeriosis in Australia. Epidemiol. Infect. 139, 437-445.

de Noordhout, C.M., Devleesschauwer, B., Angulo, F.J., Verbeke, G., Haagsma, J., Kirk, M., Havelaar, A., Speybroeck, N., 2014. The global burden of listeriosis: a systematic review and meta-analysis. Lancet Infect. Dis. 14, 1073-1082.

Desai, A.N., Anyoha, A., Madoff, L.C., Lassmann, B., 2019. Changing epidemiology of Listeria monocytogenes outbreaks, sporadic cases, and recalls globally: a review of ProMED reports from 1996 to 2018. Int. J. Infect. Dis 84, 48-53.

EFSA, ECDC, 2018. The European Union summary report on trends and sources of zoonoses, zoonotic agents and food-borne outbreaks in 2017. EFSA J. 16 (5500), 5262.

EFSA BIOHAZ Panel, Ricci, A., Allende, A., Bolton, D., Chemaly, M., Davies, R., Fernández Escámez, P., Girones, R., Herman, L., Koutsoumanis, K., Nørrung, B., Robertson, L., Ru, G., Sanaa, M., Simmons, M., Skandamis, P., Snary, E., Speybroeck, N., Ter Kuile, B., Threlfall, J., Wahlstrom, H., Takkinen, J., Wagner, M. Arcella, D., Da Silva Felicio, M., Georgiadis, M., Messens, W., Lindqvist, R., 2018. Scientific opinion on the Listeria monocytogenes contamination of ready-to-eat foods and the risk for human health in the EU. EFSA J. 16, 173.

Fritsch, L., Guillier, L., Augustin, J.-C., 2018. Next generation quantitative microbiological risk assessment: refinement of the cold smoked salmon-related listeriosis risk model by integrating genomic data. Microb. Risk Anal. 10, 20-27.

Gillespie, I.A., Mook, P., Little, C.L., Grant, K.A., McLauchlin, J., 2010. Human listeriosis in England, 2001-2007: association with neighbourhood deprivation. Euro. Surveill. $15,7-16$.

Gonzales-Barron, U., Thébault, A., Kooh, P., Watier, L., Sanaa, M., Cadavez, V., 2019. Strategy for systematic review of observational studies and meta-analysis modelling of risk factors for sporadic foodborne diseases. Microb. Risk Anal., 100082

Hoelzer, K., Pouillot, R., Dennis, S., 2012. Listeria monocytogenes growth dynamics on produce: a review of the available data for predictive modeling. Foodborne Pathog. Dis. 9, 661-673.
Jensen, A., Frederiksen, W., Gerner-Smidt, P., 1994. Risk factors for listeriosis in Denmark, 1989-1990. Scand. J. Infect. Dis. 26, 171-178.

Kvistholm Jensen, A., Simonsen, J., Ethelberg, S., 2017. Use of proton pump inhibitors and the risk of Listeriosis: a nationwide registry-based case-control study. Clin. Infect. Dis. 64, 845-851.

Maury, M.M., Bracq-Dieye, H., Huang, L., Vales, G., Lavina, M., Thouvenot, P., Disson, O., Leclercq, A., Brisse, S., Lecuit, M., 2019. Hypervirulent Listeria monocytogenes clones' adaption to mammalian gut accounts for their association with dairy products. Nat. Commun. 10, 2488.

Maury, M.M., Tsai, Y.H., Charlier, C., Touchon, M., Chenal-Francisque, V., Leclercq, A., Criscuolo, A., Gaultier, C., Roussel, S., Brisabois, A., Disson, O., Rocha, E.P.C., Brisse, S., Lecuit, M., 2016. Uncovering Listeria monocytogenes hypervirulence by harnessing its biodiversity. Nat. Genet. 48, 308-313.

Moura, A., Tourdjman, M., Leclercq, A., Hamelin, E., Laurent, E., Fredriksen, N., Van Cauteren, D., Bracq-Dieye, H., Thouvenot, P., Vales, G., Tessaud-Rita, N., Maury, M. M., Alexandru, A., Criscuolo, A., Quevillon, E., Donguy, M.P., Enouf, V., de Valk, H., Brisse, S., Lecuit, M., 2017. Real-time whole-genome sequencing for surveillance of Listeria monocytogenes, France. Emerg. Infect. Dis. 23, 1462-1470.

Nielsen, E.M., Björkman, J.T., Kiil, K., Grant, K., Dallman, T., Painset, A., Amar, C., Roussel, S., Guillier, L., Félix, B., Rotariou, O., Perez-Reche, F., Forbes, K., Strachan, N., 2017. Closing Gaps For Performing a Risk Assessment On Listeria monocytogenes in Ready-To-Eat (RTE) Foods: Activity 3, the Comparison of Isolates from Different Compartments Along the Food Chain, and from Humans Using Whole Genome Sequencing (WGS) Analysis. EFSA Supporting Publications, p. 14.

Nyarko, E., Kniel, K.E., Millner, P.D., Luo, Y., Handy, E.T., Reynnells, R., East, C., Sharma, M., 2016. Survival and growth of Listeria monocytogenes on whole cantaloupes is dependent on site of contamination and storage temperature. Int. J. Food Microbiol. 234, 65-70.

Ooi, S.T., Lorber, B., 2005. Gastroenteritis due to Listeria monocytogenes. Clin. Infect. Dis. 40, 1327-1332.

Painset, A., Björkman, J.T., Kiil, K., Guillier, L., Mariet, J.-F., Félix, B., Amar, C., Rotariu, O., Roussel, S., Perez-Reche, F., Brisse, S., Moura, A., Lecuit, M., Forbes, K., Strachan, N., Grant, K., Møller-Nielsen, E., Dallman, T.J., 2019. LiSEQ - wholegenome sequencing of a cross-sectional survey of Listeria monocytogenes in ready-toeat foods and human clinical cases in Europe. Microb. Genom. 5, e000257.

Pouillot, R., Hoelzer, K., Chen, Y., Dennis, S.B., 2015. Listeria monocytogenes dose response revisited-Incorporating adjustments for variability in strain virulence and host susceptibility. Risk Anal. 35, 90-108.

Schlech 3rd, W.F., Schlech, W.F.T., Haldane, H., Mailman, T.L., Warhuus, M., Crouse, N., Haldane, D.J., 2005. Does sporadic Listeria gastroenteritis exist? A 2-year population-based survey in Nova Scotia, Canada. Clin. Infect. Dis. 41, 778-784.

Self, J.L., 2016. Notes from the Field: Outbreak of Listeriosis Associated With Consumption of Packaged Salad—United States and Canada, 2015-2016. MMWR Morbidity and mortality weekly report 65.

Van Walle, I., Björkman, J.T., Cormican, M., Dallman, T., Mossong, J., Moura, A., Pietzka, A., Ruppitsch, W., Takkinen, J., 2018. Retrospective validation of whole genome sequencing-enhanced surveillance of listeriosis in Europe, 2010 to 2015. Eurosurveillance 23, 1700798.

Varma, J.K., Samuel, M.C., Marcus, R., Hoekstra, R.M., Medus, C., Segler, S., Anderson, B.J., Jones, T.F., Shiferaw, B., Haubert, N., Megginson, M., McCarthy, P. V., Graves, L., Gilder, T.V., Angulo, F.J., 2007. Listeria monocytogenes infection from foods prepared in a commercial establishment: a case-control study of potential sources of sporadic illness in the United States. Clin. Infect. Dis. 44, 521-528.

Viechtbauer, W., 2010. Conducting meta-analyses in R with the metafor package.201036, 48. 